Revue internationale P.M.E.

Économie et gestion de la petite et moyenne entreprise

Revue

internationale

PME

\title{
L'État et l'entrepreneur informel en Afrique
}

\section{Émile-Michel Hernandez}

Volume 10, numéro 1, 1997

URI : https://id.erudit.org/iderudit/1009019ar

DOI : https://doi.org/10.7202/1009019ar

Aller au sommaire du numéro

Éditeur(s)

Presses de l’Université du Québec

ISSN

0776-5436 (imprimé)

1918-9699 (numérique)

Découvrir la revue

Citer cette note

Hernandez, É.-M. (1997). L'État et l'entrepreneur informel en Afrique. Revue internationale P.M.E., 10(1), 103-122. https://doi.org/10.7202/1009019ar

\section{Résumé de l'article}

Aujourd'hui, en Afrique, l'importance prise par l'informel est telle que les États ne peuvent plus éviter une réflexion approfondie sur sa place dans leur économie et sur l'attitude qu'ils doivent adopter.

L'informaiité offre un certain nombre d'avantages, lesquels sont présentés dans une première partie, tandis que ses inconvénients sont analysés en deuxième partie. Face à ce phénomène et à ses contradictions, la position des États a évolué, passant en quelques années d'une attitude répressive ou indifférente à une attitude plus constructive, ce que nous verrons en troisième partie. La conclusion, enfin, propose aux États des voies d'action possibles pour faciliter le fonctionnement du secteur informel sans risquer de le perturber.
Ce document est protégé par la loi sur le droit d'auteur. L'utilisation des services d'Érudit (y compris la reproduction) est assujettie à sa politique d'utilisation que vous pouvez consulter en ligne.

https://apropos.erudit.org/fr/usagers/politique-dutilisation/ 


\title{
L'État et l'entrepreneur informel en Afrique
}

Émile-Michel HERNANDEZ

Université de Nantes et

Université du Bénin à Lomé

\section{MOTS CLÉS}

\section{Attitude de l'État - Coûts de la formalité - Coûts de l'informalité Coûts de transaction - Entrepreneur informel-Secteur informel Sous-optimisation des facteurs de production}

\begin{abstract}
RÉSUMÉ
Aujourd'hui, en Afrique, l'importance prise par l'informel est telle que les États ne peuvent plus éviter une réflexion approfondie sur sa place dans leur économie et sur l'attitude qu'ils doivent adopter.

L'informalité offre un certain nombre d'avantages, lesquels sont présentés dans une première partie, tandis que ses inconvénients sont analysés en deuxième partie. Face à ce phénomène et à ses contradictions, la position des États a évolué, passant en quelques années d'une attitude répressive ou indifférente à une attitude plus constructive, ce que nous verrons en troisième partie. La conclusion, enfin, propose aux États des voies d'action possibles pour faciliter le fonctionnement du secteur informel sans risquer de le perturber.
\end{abstract}

\section{L'AUTEUR}

Émile-Michel Hernandez est titulaire d'un doctorat en économie et administration des entreprises et d'un doctorat d'État en sciences de gestion. II est maître de conférences en sciences de gestion à l'Université de Nantes et à l'Université du Bénin à Lomé (Togo). Ses travaux de recherche et ses publications sont consacrés, d'une part, à l'entrepreneuriat et à ses spécificités en Afrique et, d'autre part, à l'adaptation du management au contexte culturel, historique et institutionnel de l'Afrique. 


\begin{abstract}
Today, in Africa, the importance of the informal sector is so great that the States can't avoid a profond thought on its place in their economy, and the policies they may carry out.

The informality presents some advantages which are analysed in the first part. But it has also drawbacks (second part). Concerning this phenomenon, and its contradictions, the States' policy has evolved in a few years from a repressive or uninterested attitude to a more constructive one (third part). Finally, the conclusion comes out with propositions to be used by States to facilitate the informal sector's life without risk of any kind of perturbation.
\end{abstract}

\title{
RESUMEN
}

Hoy día, en África, la importancia que supone lo informal es tan grande que los Estados no pueden evitar una reflexión profunda sobre la plaza que ocupa en su economía y la actitud que han de adoptar.

La informalidad supone cierto número de ventajas que se abarcarán en la primera parte. No obstante también supone ciertos inconvenientes (Véase la segunda parte). Frente a este fenómeno,y a sus contradicciones, la postura de los Estados ha evolucionado pasando en pocos anos de una actitud represiva o de indiferencia a una actitud más constructiva (Véase la tercera parte). La conclusión, por fin, propone, para los Estados, vias posibles de acción, para facilitar el funcionamiento del sector informal sin riesgo alguno de trastornarlo.

\section{Introduction}

La conférence du Caire sur la population et le développement, qui a eu lieu du 15 août au 5 septembre 1994, a attiré l'attention de l'opinion publique internationale sur la véritable explosion démographique que va connaître l'Afrique subsaharienne dans les prochaines années : 500 millions d'habitants en $1990 \ldots$ et peut-être 1300 millions vers 2025 .

Inéluctablement, les États africains feront face à de dramatiques problèmes d'emploi de leurs ressortissants. Les possibilités d'embauche du secteur qualifié de «moderne » sont excessivement limitées. Les plans d'ajustement structurel imposés par le FMI et la Banque mondiale ont, en général, des conséquences négatives pour l'emploi. Les effectifs de fonctionnaires doivent être revus à la baisse ; et les entreprises publiques liquidées, restructurées ou privatisées, toutes mesures se traduisant par des licenciements. Les Africains devront, par conséquent, continuer à se tourner vers le secteur informel comme ils ont déjà commencé à le faire depuis plusieurs années. 
Alors que les entreprises africaines modernes, publiques ou privées, connaissent de grandes difficultés, celles relevant du secteur informel résistent à la crise et même se multiplient (Hugon, 1980). Aussi, en milieu urbain, le secteur informel est-il devenu la principale source d'emplois pour les populations locales.

De nombreux chercheurs se sont intéressés à ces unités de production et ont essayé de comprendre les raisons de leur succès. L'explication essentielle qui ressort de leurs travaux est culturelle : l'entreprise informelle respecte la culture et les valeurs africaines. Pour Mamadou Dia (1992), la réussite des unités informelles, pourtant aux prises avec un environnement hostile et ne pouvant compter sur aucune aide de l'État, s'explique avant tout par «leur aptitude à concilier les valeurs sociales et culturelles de l'Afrique avec la nécessaire efficacité économique». Philippe Engelhard (1987-1988) parle d' «une adéquation qui nous échappe entre le mode d'organisation des entreprises de ce " secteur" et les caractéristiques du système culturel et du système économique environnant ». Taoufik Ben Abdallah et Philippe Engelhard (1990) indiquent que l'ensemble des systèmes et des processus économiques et sociaux du secteur informel sont «en osmose» avec les cultures ambiantes. Ces auteurs reconnaissent donc que si l'informel constitue une réponse de survie, il semble correspondre assez bien aux valeurs socioculturelles africaines.

Une autre raison de ce succès est classiquement évoquée : le bas niveau des prix pratiqués rendu possible par le non-respect des obligations sociales et fiscales. Enfin, il faut relever la mise en œuvre par l'entrepreneur informel d'un véritable marketing-mix implicite pour satisfaire les besoins de sa clientèle. Les produits proposés, les prix pratiqués, les méthodes de distribution et de communication utilisées sont parfaitement adaptés à la demande locale (Hernandez, 1995b).

Aujourd'hui, en Afrique, l'importance prise par l'informel est telle que les États ne peuvent plus se dispenser d'une réflexion approfondie sur sa place dans leur économie, et l'attitude qu'ils doivent adopter envers lui.

Le non-respect des lois présente des avantages pour les entrepreneurs, car la légalité a un coût qui fera l'objet d'une première partie. Mais l'informalité ne présente pas que des avantages; elle a aussi ses inconvénients, ses coûts, lesquels seront analysés dans une deuxième partie. Face à ce phénomène, et à ses contradictions, la position des États a évolué passant en quelques années d'une attitude répressive ou indifférente à une attitude plus constructive. C'est le sujet de la troisième partie. Enfin, la conclusion propose aux États des voies d'action possibles pour faciliter le fonctionnement du secteur informel sans risquer de le perturber. 


\section{Avantages de l'informalité}

Le respect des règles légales n'est jamais gratuit. Ses coûts sont de deux ordres : d'une part, le règlement de taxations diverses, d'autre part, le temps nécessaire au respect des formalités. Dans un pays en voie de développement (PVD), deux contraintes supplémentaires s'ajoutent : le mauvais fonctionnement des administrations locales qui augmente considérablement la durée des formalités, donc leur coût, et la nécessité parfois incontournable de verser des «pots-de-vin » pour faire avancer un dossier. Les fonctionnaires des PVD sont mal payés, voire pas payés du tout, ils ont souvent de lourdes charges familiales ; aussi, nombreux sont ceux qui monnaient leurs services, transformant ainsi leur emploi en source de rente.

Plusieurs chercheurs ont donc essayé de mesurer le coût du respect des règles légales pour des acteurs informels. Le premier, et le plus connu, est Hernando De Soto qui a mené ses travaux dans le contexte péruvien. À sa suite, d'autres auteurs, comme Sourou Joseph Attin, Ridha Ferchiou et Pierre Pigeon ont réalisé des enquêtes équivalentes dans divers pays africains.

\subsection{Les avantages de l'informalité au Pérou}

De Soto (1994a, 1994b) et son Institut Liberté et Démocratie, fondé au début des années 1980 , se sont intéressés à quatre secteurs où l'informel est particulièrement développé au Pérou: le secteur de l'industrie, celui du logement, celui du commerce et, enfin, celui du transport. Pour chacun, ils ont mesuré le coût d'accession à la légalité et le coût du maintien de l'activité dans le cadre légal : ils sont très élevés, surtout quand on les replace dans le contexte péruvien. Les résultats obtenus par De Soto vont être brièvement présentés.

\subsubsection{Coûts d'accession à la légalité}

Pendant l'été 1983, une équipe de chercheurs de l'ILD simula la création, en nom personnel, d'un petit atelier de confection dans une zone industrielle des environs de Lima. Ils conclurent qu'un individu d'un milieu modeste devait consacrer 289 jours à des démarches avant de réunir les onze conditions nécessaires à l'installation d'une petite industrie, pour un coût direct de 194,40 \$. De plus, comme presque dix mois d'attente sont nécessaires pour obtenir les autorisations, il faut ajouter un manque à gagner net de 1036,60 \$, soit un coût total de $1231 \$$, c'est-à-dire trente-deux fois le salaire minimal péruvien.

Pour le logement, un groupe de familles modestes voulant accéder à la propriété immobilière urbaine doit pour cela solliciter l'adjudication d'un terrain en friche appartenant à l'État, présenter ses projets d'aménagement, obtenir un permis de construire, et enfin un certificat de conformité des travaux réalisés. L'ensemble de ces démarches nécessite un délai de 83 mois, soit 6 ans et 
onze mois. Quant à leurs coûts, ils sont proprement exorbitants. Les seules formalités d'adjudication du terrain ont été estimées à $2156 \$$ par individu, soit 4 ans et 6 mois de salaire minimal.

L'ouverture légale d'une petite boutique nécessite de s'adresser à trois administrations différentes, soit 43 jours de démarches, pour un coût total de $591 \$$ représentant quinze fois le salaire minimal. Quant à la formalisation des marchés, c'est-à-dire la période qui s'écoule entre la formation d'un petit marché, réunion spontanée de quelques commerçants informels, et sa transformation en structure officielle, elle nécessite 17 ans en moyenne.

\subsubsection{Coûts de maintien du cadre légal}

Une fois l'activité établie dans un cadre légal, il faut l'y maintenir; ce qui engendre également des coûts. De Soto et son équipe ont étudié un échantillon d'une cinquantaine de petites entreprises industrielles employant de 1 à 4 personnes. Il en ressort que les coûts de maintien dans le cadre légal, qualifiés par cet auteur de « coûts de durabilité de la légalité », représentent $348 \%$ de leurs profits nets d'impôt et $11 \%$ de leurs coûts de production. Ils se répartissent de la façon suivante : $21,7 \%$ de coûts fiscaux, $72,7 \%$ de coûts légaux non fiscaux, et enfin $5,6 \%$ de coûts d'usage des services publics. Sur $100 \$$ d'excédent constitué, l'entrepreneur dispose seulement de 23,30 \$ et doit en consacrer 76,70\% au maintien dans le cadre légal, $17,60 \%$ pour les coûts fiscaux et $59,19 \%$ pour les coûts légaux non fiscaux. Ces derniers, qui consomment donc les trois cinquièmes de l'excédent réalisé, correspondent au respect d'obligations sociales et à celui d'innombrables contraintes administratives.

\subsection{Les avantages de l'informalité}

Plusieurs auteurs, s'inspirant du travail de De Soto, ont mené des études comparables dans divers pays africains. Dans l'ensemble, leurs conclusions rejoignent les siennes : pour un entrepreneur informel, le coût de la légalité est très élevé.

Par ailleurs, Sourou Joseph Attin (1990) s'est intéressé aux entreprises informelles béninoises. Il a étudié dans 92 entreprises exerçant divers métiers l'influence du respect des obligations sociales et fiscales sur le taux d'excédent brut d'exploitation (EBE) dégagé.

Il faut noter que, dans le contexte béninois, la baisse moyenne du taux d'EBE (31\%) est nettement plus imputable au respect des obligations sociales $(27 \%)$ qu'à celui des obligations fiscales $(4 \%)$. Ainsi, le respect des obligations légales amènerait les entrepreneurs béninois, pour maintenir leurs revenus constants, soit à augmenter leurs prix en moyenne de $31 \%$, ce qui poserait des problèmes à leur clientèle peu solvable et réduirait un peu plus la demande, soit à améliorer la productivité de leur personnel, donc à réduire leurs effectifs. 
TABlEAU 1

Coût de la légalité au Bénin

\begin{tabular}{lccc}
\hline Métiers & $\begin{array}{c}\text { Taux d'EBE sans } \\
\text { la légalité (\%) }\end{array}$ & $\begin{array}{c}\text { Taux d'EBE en } \\
\text { respectant les } \\
\text { obligations sociales } \\
\text { et fiscales (\%) }\end{array}$ & $\begin{array}{c}\text { Baisse du taux } \\
\text { d'EBE due au } \\
\text { respect de la légalité } \\
\text { (\%) }\end{array}$ \\
\hline Maçon & 85 & 56 & 29 \\
Menuisier & 78 & 39 & 49 \\
Tailleur & 88 & 65 & 23 \\
Mécanicien vélomoteur & 95 & 76 & 19 \\
Mécanicien auto & 82 & 74 & 8 \\
Soudeur & 84 & 73 & 11 \\
Ferblantier & 53 & 25 & 28 \\
Photographe & 50 & 22 & 28 \\
Restaurateur & 66 & 34 & 97 \\
Zemidjan (taxi-moto) & 35 & 62 & 25 \\
Taxi-auto & 37 & 12 & 20 \\
Commerce & 99 & 79 & $31 \%$ \\
\hline Moyenne & $71 \%$ & $40 \%$ & \\
\hline
\end{tabular}

Les travaux de Ridha Ferchiou (1990), pour leur part, concernent la Tunisie. Il a étudié 60 petites entreprises informelles du district de Tunis et a mesuré également le coût de la légalisation et son impact sur les revenus nets des entrepreneurs. Il a d'abord déterminé, par type d'activité, le revenu mensuel de l'entrepreneur. Puis, il a calculé ce que deviendrait ce revenu s'il respectait ses obligations sociales et fiscales.

TABLEAU 2

Coût de la légalité en Tunisie

\begin{tabular}{lcccc}
\hline Types d'activités & $\begin{array}{c}\text { Revenus mensuels } \\
\text { en dinar sans la } \\
\text { légalité }\end{array}$ & $\begin{array}{c}\text { Revenu mensuel } \\
\text { en dinar en } \\
\text { respectant la } \\
\text { légalité }\end{array}$ & $\begin{array}{c}\text { Exprimée en } \\
\text { dinar }\end{array}$ & $\begin{array}{c}\text { Exprimée en } \\
\text { pourcentage }\end{array}$ \\
\hline Production & 697 & 553 & -144 & $-20,66$ \\
Services & 498 & 368 & -130 & $-26,10$ \\
Petit commerce & 441 & 350 & -91 & $-20,63$ \\
Bâtiment & 647 & 349 & -298 & $-26,06$ \\
Transport & 197 & 172 & -25 & $-12,69$ \\
\hline Ensemble & & & & \\
de l'échantillon & 540 & 395 & -145 & $-26,85$ \\
\hline
\end{tabular}


Là encore, le respect de la légalité diminuerait sérieusement le revenu de ces entrepreneurs puisque, pour l'ensemble de l'échantillon, il passerait de 540 dinars à 395 dinars, soit de 4,9 fois le SMIG à 3,6 fois le SMIG. Les entrepreneurs de bâtiment seraient les plus lésés par cette législation tant en valeur relative $(-46,06 \%)$ qu'en valeur absolue $(-298$ dinars). Les transporteurs seraient les moins touchés $(-12,69 \%$ et -25 dinars), mais leur revenu ne représentant plus qu'une fois et demie le SMIG, leur survie pourrait être menacée.

Pierre Pigeon (1994), de son côté, a étudié 103 entreprises de Bangui, en Centrafrique. Il a adopté la même méthodologie qu'Attin et Ferchiou : déterminer la rentabilité de l'unité informelle et les revenus de l'entrepreneur, puis estimer ce qu'ils deviendraient en cas de respect des législations sociales et fiscales.

\section{TABLEAU 3}

\section{Coût de la légalité à Bangui (Centrafrique)}

\begin{tabular}{lcccc}
\hline Types d'activités & $\begin{array}{c}\text { Revenus mensuels } \\
\text { en milliers de } \\
\text { FCFA sans } \\
\text { la légalité }\end{array}$ & $\begin{array}{c}\text { Revenu mensuel } \\
\text { en milliers de } \\
\text { FCFA en } \\
\text { respectant } \\
\text { la légalité }\end{array}$ & $\begin{array}{c}\text { Exprimée en } \\
\text { milliers de } \\
\text { FCFA }\end{array}$ & $\begin{array}{c}\text { Exprimée en } \\
\text { pourcentage }\end{array}$ \\
\hline Production & 75 & 34 & -31 & $-41,33$ \\
Services & 49 & -4 & -53 & $-108,16$ \\
Commerce & 188 & 4 & -184 & $-97,87$ \\
Bâtiment & 82 & -44 & -38 & $-46,34$ \\
Transport & 149 & -16 & -165 & $-110,74$ \\
\hline Ensemble & & & & \\
de l'échantillon & 102 & 12 & -90 & $-88,23$ \\
\hline
\end{tabular}

Dans le contexte banguissois, la légalisation diminuerait non seulement les revenus des entrepreneurs, mais entraînerait à court terme la disparition de la plupart de ces unités avec des conséquences extrêmement dommageables pour l'environnement économique et social local. Autrement dit, même si elles le souhaitaient, les unités économiques enquêtées ne pourraient s'offrir une légalité totale. Pour Pierre Pigeon, l'informalité est imposée à la microentreprise banguissoise par «sa position structurelle défavorable (étroitesse du marché, difficulté d'accéder aux ressources) et l'inadaptation de règles juridiques héritées de l'époque coloniale». 
Ces études ${ }^{1}$ font clairement ressortir les coûts de la légalité, coûts d'accès puis de maintien. La longueur des démarches administratives, leurs prix, les charges sociales et fiscales diminueraient très nettement la rentabilité d'unités économiques déjà fragiles et même, dans certains contextes, entraîneraient leur disparition. La légalisation de l'informel, pour être économiquement réaliste, suppose donc d'importantes modifications du cadre légal. Il faut également relever que, dans l'univers informel, les exigences du court terme sont prégnantes. Si les lenteurs administratives sont déjà difficiles à admettre pour l'entrepreneur occidental, elles sont tout à fait insupportables pour son homologue des PVD.

\section{Inconvénients de l'informalité}

Ils sont plus difficiles à appréhender que ses avantages. Établir le compte de résultats d'une entreprise informelle pour déterminer sa rentabilité et le revenu de l'exploitant, puis y intégrer les dépenses supplémentaires liées au respect des lois ne présente pas de difficultés majeures. Déterminer les effets négatifs de l'informalité sur les résultats de l'entreprise est, par contre, plus délicat. Il est certes possible de repérer les facteurs qui nuisent à l'exploitation optimale de l'unité, mais il est difficile de les estimer de façon précise.

Échapper aux lois ne présente donc pas que des avantages, mais aussi des inconvénients qui vont maintenant être présentés. Une distinction sera établie entre coûts directs et indirects de l'informalité.

\subsection{Coûts directs de l'informalité}

Ce sont les plus faciles à évaluer. Ils sont de deux ordres : la taxation informelle et le coût des ressources financières.

\subsubsection{La taxation informelle}

L'illégalité du secteur autorise l'arbitraire des agents de la puissance publique. Ces exactions sont facilitées par le fait que les informels sont souvent des étrangers. Ainsi, Abidjan attire des migrants de l'ensemble du Sahel et de la Côte

1. Une autre étude, réalisée par Pascal Rutake et Ignace Ranguza sur 150 unités économiques de Bujumbura au Burundi (voir le document de recherche du BIT série micro-entreprises et secteur informel S. INF 1-4), donne des résultats tout à fait comparables. La légalisation entraînerait une réduction considérable du revenu $(48,3 \%)$ pour les gérants des unités économiques informelles. Le taux de profit moyen actuel de $61,0 \%$ tomberait à $31,5 \%$. Alors qu'il oscillait entre $38,6 \%$ et $75,0 \%$, il varierait en cas de légalisation, entre $10,4 \%$ et $42,7 \%$. 
de l'Afrique de l'Ouest, de la Guinée au Nigeria. Il est extrêmement difficile d'évaluer combien les entrepreneurs dépensent en taxes informelles. Selon Pierre Pigeon, en Centrafrique, tous les secteurs sont touchés avec une nette prédominance toutefois pour le transport. Le seul chiffre cité dans son étude concerne un vendeur de méchoui qui affirme donner quelque 5000 FCFA par semaine aux policiers corrompus, ce qui, comparé aux revenus des informels, représente une somme considérable. Dans certains cas, le niveau de prédation est tellement élevé qu'il amène les informels à cesser leurs activités ${ }^{2}$. Il faut également signaler qu'en Centrafrique le respect de la légalité n'amène pas automatiquement la tranquillité avec les pouvoirs publics puisque seulement $50 \%$ des entrepreneurs légaux n'ont jamais eu d'amende, $78 \%$ ont évité une fermeture d'établissement et $46 \%$ n'ont pas reçu de menaces.

Au Pérou, selon l'étude de De Soto (1994a), les entrepreneurs informels consacreraient 10 à $15 \%$ de leurs revenus bruts à payer des dessous de table aux autorités, ce pourcentage tombant à seulement $1 \%$ pour les petits entrepreneurs légaux.

\subsubsection{Le coût des ressources financières}

Dans les pays développés et dans le tiers monde, les banquiers sont réticents à financer les petites unités économiques, et le caractère informel des unités vient accentuer cette réticence. Les conséquences sont doubles : d'une part, le recours à l'emprunt est le plus limité possible, ce qui réduit les possibilités d'investissement et de développement ; d'autre part, lorsque c'est indispensable, l'entrepreneur s'adresse aux prêteurs informels qui pratiquent des taux très élevés.

Christian Morrison, Henri-Bernard Solignac Lecomte et Xavier Oudin (1994) ont étudié les sources de financement des microentreprises dans plusieurs pays africains. Leurs travaux font ressortir les limites de l'intervention du secteur bancaire officiel.

Les fonds utilisés dans ces petites unités proviennent essentiellement de l'entrepreneur lui-même et de son entourage familial ou amical. Le recours aux prêts officiels ou informels est réduit ; les premiers sont difficiles à obtenir et les seconds onéreux. Pourtant ces unités ont de réels besoins de financement. Lorsqu'on demande aux entrepreneurs informels de nous dire quelles ont été les principales difficultés rencontrées, l'étroitesse de la demande et le manque

2. En 1996, une Togolaise voulant ramener 80000 FCFA de bijoux du Bénin se vit réclamer 50000 FCFA par les douaniers, ce qui enlevait tout intérêt économique à l'opération. Devant le caractère exorbitant de cette demande, elle préféra leur abandonner son stock et renoncer à cette activité. 
de crédits sont les contraintes les plus souvent citées. Ainsi, dans l'étude de Ridha Ferchiou (1990) sur la Tunisie, l'exiguïté du marché et le manque d'acheteurs recueillent $43,3 \%$ des réponses et le manque de crédits, $16,7 \%$. Le crédit servirait à acheter des équipements $(53,3 \%)$, à acquérir des matières premières $(18,3 \%)$, et à financer le besoin en fonds de roulement $(1,7 \%)$. De même au Niger, selon Isabelle Joumard (1993), le manque de débouchés est cité en premier (53\%) et les problèmes financiers en second $(17,3 \%)$.

TABLEAU 4

Sources de financement des microentreprises

\begin{tabular}{|c|c|c|c|c|}
\hline Types de financement & Algérie (\%) & Tunisie (\%) & Niger (\%) & Swaziland (\%) \\
\hline $\begin{array}{l}\text { Autofinancement, } \\
\text { épargne personnelle }\end{array}$ & - & 71.5 & 793 & 770 \\
\hline Prêts famille/amis & 34,8 & 19,2 & 4,7 & 9,3 \\
\hline Dons, héritage & 17,8 & 3,4 & - & - \\
\hline Banque & 3,7 & 8,6 & 0,7 & 5,2 \\
\hline $\begin{array}{l}\text { Aide publique, crédit } \\
\text { "Emploi des jeunes " } \\
\text { Épargne collective }\end{array}$ & 0,7 & 0,3 & 0,7 & 2,1 \\
\hline informelle, prêts sur gages & 0,7 & 1,4 & - & 4,5 \\
\hline Autres & 7,4 & - & 0,0 & 0,0 \\
\hline Total & 65,1 & 104,4 & 100,1 & 98,1 \\
\hline
\end{tabular}

Note : Au Niger dans la catégorie « Autres » sont aussi compris les dons de la famille et l'héritage.

De nombreux chercheurs se sont intéressés à la finance informelle; ils en ont montré la souplesse et l'inventivité. Si elle a un rôle social évident, il est plus limité en matière entrepreneuriale, comme le montre d'ailleurs l'étude de Morrison, Solignac Lecomte et Oudin. Elle présente en effet deux limites : elle est orientée principalement sur le court terme et peut difficilement servir à financer des investissements à moyen ou à long terme, et elle est très coûteuse. Les taux pratiqués sont variables, fonctions de la personnalité de l'emprunteur et de l'usage qu'il veut faire des sommes empruntées. La proximité familiale ou amicale les fait diminuer, la rentabilité de l'utilisation projetée les fait monter. À titre indicatif, en Côte-d'Ivoire, pour 1000 FCFA empruntés le débiteur doit rembourser 1200 FCFA en fin de mois (20\%) et 2000 FCFA en fin d'année (100\%). Pour le Pérou, selon De Soto, les taux d'intérêts sur le marché informel du crédit à Lima étaient de $22 \%$ par mois en juin 1985, alors qu'ils atteignaient un maximum de $4,9 \%$ sur le marché bancaire. Ces taux rendent le recours au crédit très coûteux. Et on comprend aisément que, dans la mesure du possible, l'entrepreneur informel préfère s'en passer et recourir à l'autofinancement. 


\subsection{Coûts indirects de l'informalité}

Ces coûts sont beaucoup plus difficiles à appréhender, d'abord, et à quantifier ensuite. La notion de coût de transaction sera utilisée pour les expliciter. Elle a été introduite par Ronald H. Coase en 1937 (1987), développée par Oliver E. Williamson (1994a, 1994b) et commentée par divers auteurs, comme Olivier Favereau (1988), Xavier Gillis (1992), Patrick Joffre (1989) et Michel Marchesnay (1991). Un entrepreneur, selon Coase, souhaite internaliser toute transaction que le marché lui assurerait dans des conditions plus onéreuses. C'est ce qui explique le développement des organisations et sa limite : « une entreprise tendra à s'agrandir jusqu'à ce que les coûts d'organisation de transactions supplémentaires en son sein deviennent égaux au coût de réalisation de cette même transaction par le biais d'un échange sur le marché, ou aux coûts d'organisation dans une autre entreprise ». Un des objectifs de l'entrepreneur, qu'il soit officiel ou informel, est de minimiser ses coûts de transaction. Michel Marchesnay précise cette notion : «Le coût de transaction ne comprend donc pas que le coût relatif du produit (selon une confusion fréquente). Il comprend des coûts ex ante (signature, négociation, sauvegarde du contrat) et ex post (révision et fonctionnement du contrat). » Dans son ouvrage sur Les institutions de l'économie, Oliver E. Williamson (1994a) indique que : «Les coûts de transaction constituent l'équivalent économique des frictions dans les systèmes physiques. » Leur étude peut être qualifiée, par analogie, de tribologie économique. Pour Patrick Joffre (1989), le coût de transaction désigne le prix du face à face entre deux agents économiques, individuels ou collectifs. Il intègre les ressources utilisées pour négocier et suivre le contrat portant transfert des droits de propriété d'un individu à un autre, d'une organisation à une autre : «Ces ressources comprennent notamment le travail nécessaire à la recherche d'un compromis, les efforts de standardisation et de certification de la qualité des biens échangés ; les honoraires des conseils chargés de s'assurer du caractère légal des dispositions envisagées et, enfin, les impôts attachés à certains types de transactions. »

Le domaine de l'informel est celui de l'incertain. Les coûts engendrés par l'incertitude et la crainte de voir le partenaire ne pas respecter ses engagements ou tirer parti d'un rapport de force sont maximisés. La sécurité contractuelle est inexistante. Les contrats légalement exigibles rendent crédibles les promesses faites et incitent les parties à ne prendre que les engagements qu'elles sont en mesure de respecter. Ils peuvent même inclure une clause pénale condamnant à une compensation la partie qui déclare forfait. Lorsque la justice fonctionne, un système légal assurant à tous un accès aisé aux tribunaux facilite les transactions entre individus. Toutes ces garanties n'existent pas dans le secteur informel ; aussi, l'entrepreneur doit-il y jouer un rôle que Janet T. Landa (1993) qualifie de « combleur de vide » face à l'insécurité contractuelle qui règne dans les pays en voie de développement. 
En quoi consiste ce rôle ? «Il s'agit notamment de la capacité du commerçant à créer un groupe ethniquement homogène et des réseaux d'échanges personnalisés en vue de réduire le coût inhérent au respect des contrats. » Cela aura deux conséquences. La première est que l'entrepreneur préférera entretenir des relations commerciales avec les membres de son ethnie (Janet T. Landa parle de «réseau ethnique »), avec des parents, avec des proches plutôt qu'avec des étrangers: "plus la distance sociale est réduite et plus on se sent en confiance; plus on se sent en confiance et moins il en coûte de faire respecter les contrats ». La seconde est que l'entrepreneur va consacrer beaucoup de temps à développer un réseau de relations fiables. Cela limite donc les possibilités de développement de son champ d'action et de son entreprise, et entrave le développement de vastes marchés efficients.

Le réseau particulariste ethnico-familial, forme d'organisation dominante dans le Sud, constitue un quatrième mode d'organisation économique dans lequel un rapport de confiance réciproque relie les parties contractantes. Il vient compléter les trois types différents d'institutions ou modes d'organisation économique permettant, dans les économies capitalistes développées, la coordination des activités d'entreprises interdépendantes identifiées par Oliver E. Williamson : le marché et son corollaire, le contrat, l'entreprise verticalement concentrée et les relations contractuelles suivies.

L'insécurité contractuelle a un autre effet : elle oblige les entrepreneurs, pour limiter leurs risques, à diversifier leurs sources d'approvisionnement et leurs marchés bien plus qu'il ne serait nécessaire dans un cadre légal. Ils doivent donc, très souvent, travailler dans des conditions sous-optimales. De Soto fournit l'exemple d'un tailleur informel qui, au lieu de commander 1000 fermetures à glissière à un seul fournisseur, en achète 200 à cinq fournisseurs différents dont la production sera inférieure à leur seuil optimal, et qui verront leur coût à l'unité s'élever et leurs possibilités d'employer de la main-d'œuvre réduites en proportion. Même l'instauration de bonnes relations commerciales n'élimine pas totalement les risques en l'absence de sécurité contractuelle, légale et judiciaire. Ainsi, pour reprendre l'exemple précédent, le fournisseur peut ne pas effectuer la dernière livraison, même s'il a été payé, s'il estime qu'il n'y aura pas d'autres commandes à l'avenir. Quant au tailleur, il peut ne pas payer la dernière livraison s'il ne juge plus nécessaire de préserver sa réputation auprès d'un fournisseur.

Ainsi la légalité a un coût, mais l'informalité aussi : pour le personnel sans protection sociale, pour les organismes sociaux qui ne perçoivent pas de cotisations, pour les pouvoirs publics locaux et nationaux qui perdent des recettes fiscales et, enfin, pour l'entrepreneur lui-même. Il est victime d'innombrables taxations illégales, il lui est difficile de trouver du financement avec des taux d'intérêt raisonnables, il opère dans un univers incertain où de nombreuses 
contraintes gênent la réduction de coûts de transaction souvent élevés, ce qui l'amène à travailler dans des conditions de production sous-optimales.

\section{L'État et le secteur informel}

L'attitude de l'État face au secteur informel a évolué avec les années. D'abord, essentiellement répressive, elle se caractérise plus souvent aujourd'hui par le souci de définir des politiques d'intervention efficaces.

\subsection{De la répression à l'aide}

L'attitude de l'État est souvent ambiguë. Conscients du rôle de soupape de sécurité de ce secteur qui limite les risques d'explosion sociale urbaine, les pouvoirs publics alternent périodes de laisser-aller et de répression. Si le secteur informel est toléré, il reste l'objet d'une méfiance qui débouche, ici et là, sur des tentatives d'actions régulatrices sinon souvent répressives de la part des autorités nationales et de leurs experts en développement attitrés. On peut distinguer plusieurs attitudes possibles à l'égard du secteur informel.

L'attitude répressive : le secteur informel est considéré comme un frein à la modernisation des économies africaines ; il faut limiter son expansion, voire le faire disparaître. La faible rémunération de la main-d'œuvre comporte deux inconvénients : 1) elle n'incite pas les entrepreneurs à optimiser son utilisation, d'où une productivité stagnante ; 2) elle limite la demande intérieure, ce qui bloque le développement industriel local. Diverses mesures sont alors adoptées : un contrôle strict des migrations et des installations d'établissements artisanaux et commerciaux, l'obligation de respecter la législation et la multiplication des contrôles par les services de l'État, l'augmentation de la fiscalité et des contrôles fiscaux, etc. L'économie informelle ne disparâit pas. Mais pour échapper aux tracasseries administratives et financières, elle devient souterraine, au détriment de ses employés, de ses clients et de l'État.

Une deuxième attitude possible est le laisser-faire, qui a, lui aussi, ses partisans. Malcom Harper (1991) fut une fois interrogé par un groupe de fonctionnaires et d'universitaires venant d'un certain nombre de pays pauvres : «Que pouvons-nous faire pour les entrepreneurs de nos pays ? Ils trouvèrent la réponse "Laissez-les tranquilles" un peu désagréable, mais c'est encore la meilleure prescription. Les aides financières présentent le principal inconvénient, dans les pays du tiers monde, de risquer d'être détournées ou d'être attribuées plus en fonction de considérations de clientélisme, de solidarités ethniques ou amicales qu'en réponse à des critères d'efficacité potentielle. En outre, la formation présente le risque de ne pas convenir, d'être une simple transposition de techniques occidentales et d'entraîner une remise en cause 
brutale des méthodes de gestion adaptées au contexte et aux contraintes locales ; elle serait alors plus nuisible qu'utile. Les activités informelles urbaines sont le prolongement de la société traditionnelle. Une tentative d'évolution trop rapide se solderait inévitablement par un échec.

À partir de 1986, les conséquences sociales des plans d'ajustement structurel (PAS) imposés par le FMI et la Banque mondiale ont amené les gouvernements africains à s'intéresser à l'informel comme solution possible aux difficultés rencontrées. On est passé de la répression ou du laisser-faire à des politiques d'intervention visant à favoriser le développement du secteur informel. Les actions mises en œuvre concernent principalement la formation des entrepreneurs, leur regroupement et le financement de leurs projets ( $c f$. Arellano, Gasse et Verna [1992] et Charmes [1989]).

- La formation revêt un triple aspect. D'abord, elle vise l'alphabétisation, car de nombreux informels sont illettrés, surtout les femmes. Bien que cela ne les empêche pas de savoir compter, c'est un handicap important pour la bonne marche de leurs affaires. Ensuite, la formation technologique, qui doit cependant être adaptée au niveau local et ne pas être la simple transposition de données occidentales totalement inutiles dans un PVD. Enfin, c'est la formation à la gestion qui se limite le plus souvent à une initiation à la comptabilité générale en partie double paraissant totalement ésotérique aux informels, alors que des méthodes simples pour déterminer leurs prix de revient seraient plus utiles.

- Le regroupement prend souvent la forme de coopératives. Il a pour objectif de faciliter la défense des informels et de promouvoir des actions à caractère collectif visant, par exemple, à améliorer la commercialisation des produits (présence à des foires, notamment). Leur individualisme rend les regroupements difficiles, et les structures pérennes sont rares. De plus, les coopératives donnent souvent lieu à des malversations, ce qui accroît encore la méfiance des entrepreneurs et ne les incite pas à se réunir.

- Le financement, enfin, est un domaine classique d'intervention gouvernementale. La première difficulté qui se pose est de bien cibler les bénéficiaires de ce crédit pour qu'il soit utilisé à bon escient. La seconde est d'obtenir le remboursement des sommes prêtées, ce qui se fait beaucoup mieux en s'appuyant sur la pression communautaire que sur des dispositifs d'ordre juridique comme le cautionnement, le nantissement ou l'hypothèque. Jacques Charmes (1989) relève plusieurs dangers de ces politiques de crédit à la petite entreprise. La complexité des dossiers à présenter élimine la candidature de la plupart des informels au profit d'une frange supérieure du secteur. Jugeant plus rentable d'emprunter 
à des taux intéressants, elle réduira son apport personnel au minimum requis pour consacrer son épargne à l'achat de biens de consommation ou à la réalisation d'opérations immobilières. La concentration des projets dans certains secteurs jusqu'à saturation totale rend aléatoire la rentabilisation des investissements financés ( $c f$. l'exemple de la boulangerie dans de nombreux pays). Enfin, l'élévation de l'intensité capitalistique des projets va de pair avec une tendance au suréquipement ( $c f$. l'exemple du menuisier achetant des combinés à six opérations ou plus, quand seules deux opérations sont strictement nécessaires).

\subsection{Action institutionnelle et théorie des droits de propriété}

\subsubsection{L'action institutionnelle}

Les diverses actions en faveur du secteur informel qui viennent d'être présentées s'appliquent dans un cadre institutionnel fixe. L'étape suivante consiste à en préconiser une modification comme solution aux problèmes de l'informalité. C'est De Soto qui, le premier, s'est engagé dans cette voie. Pour ce chercheur, si les informels ne respectent pas les lois, c'est qu'elles sont mauvaises; il faut donc les changer. L'illégalité, c'est-à-dire le non-respect des lois officielles, ne doit pas être confondue avec l'absence totale de règles. Les informels ont établi leurs propres règles dont il faudra s'inspirer pour mieux harmoniser légalité et pratique des acteurs. Pour De Soto, l'informel, c'est la discrimination par le droit ; c'est le non-accès au droit officiel qui oblige les pauvres à ne pas le respecter et à élaborer leurs propres règles. Son influence politique lui a permis de mettre en application ses théories. Ainsi, une modification de la loi péruvienne a ramené de 289 jours à un seul la durée nécessaire à l'enregistrement d'une société; de plus, il se fait maintenant en un lieu unique et en payant une taxe unique. Depuis cette réforme, en septembre 1990, jusqu'en décembre 1994, 340000 entreprises nouvelles ont été enregistrées au Pérou. De même, l'élaboration d'un code de la propriété, fondé sur la codification du droit indigène, a permis l'enregistrement et l'attribution de titres pour 150000 parcelles, en zones urbaine et rurale, de manière rapide et pour un coût de $11,30 \$$ la parcelle au lieu de $17500 \$$. Pour ce chercheur, le cadre institutionnel étant responsable de l'informalité, sa modification en entraînera de facto la disparition.

Ces théories ont soulevé un certain scepticisme parmi les chercheurs s'intéressant à l'informel dans le contexte africain. Ainsi Pierre Pigeon (1994) a intitulé son étude «Microentreprises des pays en développement : l'illusion institutionnelle ». Pour lui, les effets de la légalisation sur le fonctionnement et la dynamique des unités économiques présenteraient moins d'avantages que d'inconvénients : "C'est une illusion de croire que la législation ou l'adaptation des lois et des règlements aux conditions du circuit informel confère des 
avantages conséquents. Une action centrée sur le cadre juridico-administratif reste extrêmement limitée. » Le principal problème du circuit informel se situe ailleurs, notamment dans la structure des marchés et dans l'insuffisance de la demande. Il est aujourd'hui renforcé par le contexte de l'ajustement structurel.

Le travail de Morrisson, Solignac Lecomte et Oudin (1994) sur les microentreprises et le cadre institutionnel de sept pays (Algérie, Équateur, Jamaïque, Niger, Swaziland, Thaïlande, Tunisie) viennent également relativiser dans une large mesure la thèse soutenue par De Soto. Qu'il s'agisse des difficultés rencontrées lors de la création de leur entreprise ou plus tard, les entrepreneurs ne citent jamais en premier les contraintes institutionnelles, mais l'instabilité ou l'insuffisance de la demande et les questions de financement. On retrouve cet ordre dans les sept pays alors que les réglementations et les niveaux de développement y sont différents. Pour ces auteurs, il est illusoire de croire qu'il suffit de changer ou de supprimer des réglementations pour assurer le développement des microentreprises. Enfin Arellano, Gasse et Verna (1992) donnent quelques recommandations concernant l'adaptation des législations nationales aux exigences de l'informel : essayer d'introduire de la flexibilité dans les systèmes légaux plutôt que chercher à les abolir, assouplir la bureaucratie presque toujours trop lourde et la surréglementation, alléger le système de taxation. Mais, pour ces auteurs également, la voie institutionnelle ne constitue qu'une possibilité d'action parmi d'autres.

\subsubsection{La théorie des droits de propriété}

Même si les thèses de De Soto peuvent soulever un certain scepticisme, elles ont le mérite de rappeler l'importance du droit comme régulateur essentiel de l'activité économique. La théorie des droits de propriété (property right) ( $c f$. Couret, 1987 et 1992; Lepage, 1978 ; Tartarin, 1982) se fonde sur l'idée simple que l'existence de droits de propriété bien déterminés est une condition essentielle à l'initiative individuelle, la maximisation de chaque utilité individuelle entraînant un accroissement de l'efficacité collective. Si elle a été largement utilisée pour analyser le développement du monde occidental, son emploi pour étudier la situation des PVD est plus récent.

Pour l'Afrique, Mamadou Koulibaly (1992), dans son ouvrage Le libéralisme. Nouveau départ pour l'Afrique Noire, se réfère à cette théorie pour expliquer le sous-développement. En Afrique, les droits de propriété, qu'ils soient administrés ou publics, sont flous, mal définis : «Le sous-développement, c'est l'imprécision des droits de propriété. Une économie sous-développée est une économie où les droit de propriété sont diffus et flous. Le développement, c'est la précision des droits de propriétés. » Pour cet auteur, quand on soutient que l'agriculture est la base et le moteur de l'industrialisation, il ne s'agit pas de 
n'importe laquelle, mais d'une agriculture fondée sur la propriété privée de la terre : «Le développement agricole passe par le développement des droits de propriété privée sur le sol, en tant que facteur de production et élément du patrimoine individuel.»

Le sous-développement a d'abord été attribué à la pénurie de capitaux et au niveau de formation insuffisant des populations, puis, plus récemment, à des contingences culturelles. Cette nouvelle approche offre d'intéressantes perspectives en relevant l'importance du droit comme technique d'organisation sociale pouvant favoriser la croissance, comme en Occident.

\section{Conclusion}

Aujourd'hui, les États d'Afrique subsaharienne ont perdu deux illusions : faire disparaître l'informel au profit d'un développement économique de type occidental ; y voir un vivier d'où émergeront les futures entreprises modernes (seul un petit nombre de ces unités est modernisable). L'informel représente environ $60 \%$ des emplois urbains, et c'est une réalité durable. Les gouvernements doivent en tenir compte et, essayer, dans la mesure du possible, d'aider toutes ces petites organisations; cette action comporte deux dimensions.

La première consiste à améliorer le fonctionnement interne des entreprises informelles. Même si certaines pratiques de gestion peuvent a priori surprendre un observateur imprégné d'ethnocentrisme, on doit reconnaître à ces acteurs un comportement rationnel (Hernandez, 1995a, 1996). Ce n'est certes pas la rationalité absolue de l'homo economicus mais la rationalité procédurale d'acteurs exerçant leur capacité de choix en situation, tenant compte d'une pluralité de contraintes et de critères, d'avantages et d'inconvénients : une éco-rationalité. Et il faut cesser de vouloir appliquer à l'Afrique en général et, a fortiori, dans ses petites unités, des modèles de gestion conçus essentiellement pour de grandes organisations occidentales. Comme l'entrepreneur informel intègre dans son comportement préoccupations sociales et économiques, un modèle de management éco-social conviendrait mieux pour en tenir compte. Si l'économie du développement constitue depuis longtemps un champ reconnu des sciences économiques, le «management du développement » reste encore largement à construire tant les chercheurs en sciences de gestion se sont, jusqu'à ce jour, peu intéressés aux spécificités des organisations dans les pays en voie de développement.

La seconde dimension est externe et porte sur l'environnement dans lequel ces unités opèrent. La plupart des États d'Afrique subsaharienne ont deux caractéristiques essentielles. D'une part, ils sont très interventionnistes sur le plan économique (législation importante et souvent tatillonne, multitude 
d'entreprises publiques et parapubliques) et, d'autre part, ils sont faibles (la législation est peu respectée, la corruption élevée, les fraudes nombreuses et l'autorité de l'État souvent très théorique). Pour tenir compte de ce contexte, l'action préconisée est modeste. Son objectif est de faciliter la vie à ce secteur, sans vouloir à tout prix le moderniser, c'est-à-dire, en fait, lui imposer les normes occidentales.

La voie institutionnelle constitue une première possibilité d'action. En Afrique francophone, l'essentiel de la législation date d'une époque où un décret d'application étendait «aux colonies » des textes de loi conçus initialement pour la France. Des textes plus simples, mieux adaptés au contexte socioéconomique de pays en voie de développement, constitueraient un cadre juridique plus réaliste et, par conséquent, plus susceptible d'être respecté par les acteurs.

La deuxième possibilité d'action concerne les agents de la puissance publique. Un paiement régulier de leurs salaires et un meilleur contrôle de leurs actions devraient permettre de maintenir la corruption dans des limites raisonnables (sa disparition restant un objectif à terme). Aujourd'hui, dans certains pays africains (comme le Zaïre, par exemple), le degré de malversation est tel que les politologues n'hésitent plus à les qualifier d' «États mafieux ».

La troisième possibilité d'action concerne l'urbanisme. Il faudrait permettre aux entrepreneurs informels de devenir propriétaires, moyennant le paiement d'un loyer modeste pendant quelques années, de terrains sommairement viabilisés, avec l'eau et l'électricité, correctement situés et légalement cessibles, où ils pourraient installer leurs ateliers ou leurs boutiques. Cette reconnaissance de leurs droits de propriété sur leurs locaux leur permettrait de travailler tranquillement sans être menacés constamment de « déguerpissement », comme c'est souvent le cas actuellement dans les grandes villes africaines. Elle constituerait également le début d'un processus capitaliste d'accumulation.

Ces mesures, certes, limitées mais suffisamment tempérées pour ne pas risquer de faire plus de mal que de bien, faciliteraient l'activité d'un secteur qui, s'il ne constitue pas un remède économique miracle pour l'Afrique, permet tout de même la survie d'une part importante de la population urbaine qui n'a actuellement aucune autre solution envisageable, et sert probablement à éviter de violentes explosions sociales. 


\section{Bibliographie}

AREllano, R., Y. GaSSE et G. Verna (1992), «Le monde de l'entreprise informelle : intervention ou laissez-faire », Centre Sahel Université Laval, Canada, Série Dossiers, $n^{\circ} 26$, septembre.

ATTIN, S. J. (1990), « Perspectives du passage du secteur informel au secteur formel au Bénin (Coût de la légalité) », Bureau international du travail, Genève, Document de travail, Série WEP 2-19/ WP.54, juillet.

Ben AbDallah, T. et P. EngelhaRd (1990), «Quel avenir pour l'économie populaire en Afrique?», Revue Quid Pro Quo, Bruxelles, vol. 1, nos 3-4, p. 14-17.

ChARMES, J. (1989), «Quelles politiques publiques face au secteur informel ?», Caisse française de développement - division des études générales, Paris, Notes et Études, n 23, avril.

COASE, R. H. (1987), «La nature de la firme », Revue Française d'Économie, Paris, hiver, p. 133-163.

COURET, A. (1987), «La théorie des droits de propriété (property rights) », dans De nouvelles théories pour gérer l'entreprise, Paris, Economica, p. 58-78.

Couret, A. (1992), «Propriété et gestion de l'entreprise », dans A. Labourdette (éd.), Mélanges en l'honneur de Jean-Guy Merigot, Paris, Economica, p. 605-617.

DE Soto, H. (1994a), L'autre sentier. La révolution informelle dans le tiers monde, Paris, Éditions La Découverte.

De Soto, H. (1994b), «L'économie informelle avenir du tiers monde?», Revue Alternatives Économiques, Paris, $\mathrm{n}^{\circ} 122$, p. 42-44.

DiA, M. (1992), «Développement et valeurs culturelles en Afrique subsaharienne », Problèmes Économiques, Paris, La Documentation Française, $\mathrm{n}^{0} 2.281,24$ juin.

ENGELHARD, P. (1987-1988), «Quelques remarques sur l'article de Ali El Idrissi ou l'économie d'Adam Smith sous les tropiques », dans Annales du Laboratoire de Gestion et Cultures, IAE de Nice, p. 51-59.

FaVereau, O. (1988), «Organisation et marché », Revue Française d'Économie, Paris, décembre, p. 65-96.

FERCHIOU, R. (1990), «Microentreprises du secteur informel à Tunis. Obstacles de caractère légal et institutionnel ", Bureau international du travail, Genève, Document de travail, Série WEP 2-19/WP.51.

GILlis, X. (1992), «Ronald H. COASE II La "nature de la firme" et la théorie des coûts de transaction », Paris, Problèmes Économiques, n 2.268, p. 7-10.

HARPER, M. (1991), «Enterprise development in poorer nations », Entrepreneurship Theory and Practice, Baylor University USA, vol. 15, n² 4, été, p. 7-11. 
HERNANDEZ, E.-M. (1995a), «La notion de gestion dans le secteur informel », Revue Française de Gestion, Paris, ${ }^{0} 103$, mars-avril-mai, p. 66-75.

HERNANDEZ, E.-M. (1995b), «L'entrepreneur informel africain et la démarche marketing », Revue Recherche et Applications en Marketing, Paris, vol. X, $\mathrm{n}^{0} 3$, p. 47-61.

HERNANDEZ, E.-M. (1996), Le management des entreprises africaines. Essai de management du développement, Paris, Éditions L'Harmattan.

Hugon, P. (1980), «Dualisme sectoriel ou soumission des formes de production au capital. Peut-on dépasser le débat?», Revue Tiers Monde, Paris, vol. XXI, $\mathrm{n}^{\circ} 82$, p. 235-259.

JOFFRE, P. (1989), «Entreprise et marché », dans Encyclopédie de Gestion, Paris, Economica, p. 994-1011.

JOUMARD, I. (1993), «Impacts des réglementations sur les petites et microentreprises : une comparaison régionale ", dans Les Orientations nouvelles de l'aide en faveur des microentreprises, Paris, OCDE, p. 49-60.

Koulibaly, M. (1992), Le libéralisme. Nouveau départ pour l'Afrique Noire, Paris, Éditions L'Harmattan/Collection Afrique 2000.

LANDA, J.T. (1993), «Culture et activité entrepreneuriale dans les pays en développement: le réseau ethnique, organisation économique », dans Brigitte Berger et al., Esprit d'entreprise, Cultures et Sociétés, Paris, Éditions Maxima, p. 195-217.

LePage, H. (1978), Demain le capitalisme, Paris, Le Livre de Poche, Collection Pluriel.

MARCHESNAY, M. (1991), «La transaction, outil d'analyse stratégique ?», dans Identités de la gestion. Mélanges en l'honneur du Professeur Pierre Lasségue, Paris, Vuibert Gestion, p. 135-146.

Morrisson C., H.-B. SolignaC LeCOMTE et X. OUdin (1994), « Microentreprises et cadre institutionnel dans les pays en développement », Paris, Études du Centre de Développement, OCDE.

Pigeon, P. (1994), «Microentreprises des pays en développement l'illusion institutionnelle », UREF / AUPELF, Notes de recherche du réseau entepreneuriat, $\mathrm{n}^{\circ}$ 94-45.

TARTARIN, R. (1982), «La théorie des droits de propriété : vers un historicisme libéral ?», dans Andreff, Cot, Frydman, Gillard, Michon et Tartarin, L'économie fiction. Contre les nouveaux économistes, Paris, Éditions François Maspero, p. 105-135.

Williamson, O.E. (1994a), Les institutions de l'économie, Paris, Inter-Éditions.

WILLIAMSON, O.E. (1994b), «Williamson et la nouvelle économie institutionnelle», Revue Alternatives Économiques, Paris, nº 122, décembre, p. 58-61. 